\title{
PREVALÊNCIA DO ALEITAMENTO MATERNO NAS CAPITAIS BRASILERAS
}

\author{
Maria Cristina ferreira Sena, Eduardo freitas da Silva, Maurício Gomes Pereira* \\ Trabalho realizado na Universidade de Brasília, Faculdade de Ciências da Saúde, Programa de Pós-Graduação em Ciências da Saúde e na Fundação de \\ Ensino e Pesquisa em Ciência da Saúde, Brasília, DF
}

\author{
*Correspondência \\ SHIS QI 15 conjunto 5 \\ casa 20 - Lago Sul - \\ Brasília - DF \\ Cep 71635-250 \\ Tel.: (61) 3364-0552/ \\ (61) $8119-0355$ \\ mauriciogpereira@gmail.com
}

RESUMO

Oвjetivo. Estimar a prevalência do aleitamento materno e do aleitamento exclusivo para as capitais brasileiras, para as grandes regiões e para o Brasil, nas idades de 30, 120 e 180 dias, preconizadas por consenso entre especialistas para unificar as estatísticas.

Métodos. Procedeu-se a reanálise dos dados do inquérito populacional de aleitamento materno realizado em 25 capitais e no Distrito Federal, em 16 de outubro de 1999, Dia Nacional de Vacinação Infantil. A amostra probabilística do presente estudo refere-se a 10.778 crianças distribuídas nas idades mencionadas. As prevalências por ponto e por intervalo (intervalo de confiança de 95\%) foram determinadas para as capitais e então extrapoladas para as regiões brasileiras e para o Brasil. Utilizou-se a análise de regressão com o programa estatístico SAS.

Resultados. As prevalências estimadas de aleitamento materno para o Brasil foram aos 30, 120 e 180 dias, respectivamente, $87,3 \%(86,8$ - 87,7), 77,5\% (77,1 - 78,0) e 68,6\% (68,2 - 69,1) e, do aleitamento materno exclusivo, nas mesmas idades, 47,5\% (46,4 - 48,5), 17,7\% (17,2 - 18,3) e 7,7\% (7,2 - 8,2). Nas capitais, a variação da freqüência do aleitamento materno exclusivo aos 30 dias foi ampla, oscilando entre 73,4\% (Fortaleza) e 25,2\% (Cuiabá). Aos 180 dias de vida, as taxas alternaram de 16,9\%, em Belém a 2,8\%, em Cuiabá.

Conclusão. No primeiro semestre de vida houve redução moderada da prevalência do aleitamento materno e queda acentuada da prevalência do aleitamento materno exclusivo. Foram observadas diferenças importantes na freqüência do aleitamento materno exclusivo entre as capitais pesquisadas.

Unitermos: Aleitamento materno. Aleitamento materno exclusivo. Estudos transversais. Epidemiologia. Brasil.

\section{INTRODUÇÃo}

A alimentação da criança nos primeiros seis meses de vida deve estar restrita ao leite materno ${ }^{1,2}$. Inquéritos periódicos informam sobre a situação e fornecem base para ação ${ }^{3}$. A comparação dos resultados desses inquéritos enfrenta dificuldades de ordem metodológica, principalmente por falta de uniformidade nas idades utilizadas para estimar as prevalências ${ }^{4,5,6,7}$. Recentemente, foram definidas as idades de corte de 30, 120 e 180 dias para padronizar as estatísticas de aleitamento materno no país ${ }^{8}$. No inquérito nacional de 1999, específico de aleitamento materno, efetuado nas capitais brasileiras e que foi coordenado por um dos autores do presente artigo (MCFS), os resultados não contemplam essas idades ${ }^{7}$. Além disso, só foram divulgados na forma de relatório ${ }^{7}$. 0 presente trabalho apresenta o produto da reanálise da pesquisa nacional expressando os resultados como preconizado.

\section{Métodos}

\section{Características da pesquisa original}

Trata-se de um inquérito transversal, realizado no dia da Campanha Nacional de Vacinação nas capitais brasileiras e no Distrito Federal, em 16 de outubro de 19997. O Rio de Janeiro não foi incluído, pois seus técnicos justificaram ter feito pesquisa sobre o tema havia pouco tempo. De cada localidade foi selecionada amostra probabilística em duas etapas. Na primeira, obtiveram-se amostras aleatórias simples de postos de vacinação a partir das listas fornecidas pelos coordenadores locais. Na segunda etapa, que corresponde à seleção de crianças menores de um ano nos postos sorteados, empregou-se amostragem sistemática. Pessoas adequadamente treinadas realizaram entrevistas com as mães das crianças selecionadas para o estudo. As informações foram registradas em questionários padronizados e pré-testados. Também houve cuidado no planejamento de outros aspectos para controlar o erro de aferição, como exemplos a supervisão do trabalho de campo, a adequada relação entre o número de supervisores e de entrevistadores com o de mães entrevistadas, a realização da coleta de dados em um único dia e o desenvolvimento de software específico para a digitação dos dados do inquérito.

Sobre a alimentação da criança explorou-se o consumo, nas últimas 24 horas, dos seguintes itens: leite materno, água, chá, sucos, outro leite, frutas, sopas e refeição da família. As variáveis dependentes medidas foram o aleitamento materno e o aleitamento materno exclusivo e a independente a idade da criança.

\section{Reanálise dos dados}

Limitamos a presente análise a todas as crianças nas idades mencionadas, em um total de 10.778, sendo 1.259 com 30 dias de idade (abrangendo crianças entre 15 e 45 dias), 4.632 com 120 dias de idade (entre 106 e 135 dias) e 4.887 com 180 dias de idade (entre 166 e 195 dias).

Os critérios utilizados para definir o tipo de aleitamento são os estabelecidos pela Organização Mundial da Saúde?. Na categoria aleitamento materno, a condição essencial é a criança ser alimentada com o leite materno, independentemente de receber outros tipos 
SENA MCF ET AL.

Tabela I- Prevalência (\%) de aleitamento materno, por idade da criança, nas capitais, Distrito Federal, grandes regiões e estimativa para o Brasil, I999

\begin{tabular}{|c|c|c|c|c|c|c|c|c|c|}
\hline \multirow[t]{3}{*}{ Localidades* } & \multicolumn{9}{|c|}{ Idade da criança em dias } \\
\hline & \multicolumn{3}{|c|}{30} & \multicolumn{3}{|c|}{120} & \multicolumn{3}{|c|}{180} \\
\hline & $\%$ & $1 \mathrm{C} 95 \%{ }^{\dagger}$ & $\operatorname{ordem}^{\ddagger}$ & $\%$ & IC $95 \%^{\dagger}$ & ordem $^{\ddagger}$ & $\%$ & IC $95 \%^{\dagger}$ & ordem ${ }^{\ddagger}$ \\
\hline Região Norte & 90,9 & $90,0-91,7$ &. & 83,7 & $82,8-84,5$ & . & 76,7 & $75,8-77,5$ &. \\
\hline Porto Velho & 88,8 & $86,3-91,0$ & 10 & 81,2 & $78,7-83,4$ & 10 & 74,1 & $71,8-76,2$ & 8 \\
\hline Rio Branco & 85,0 & $82,0-87,6$ & 14 & 75,9 & $73,3-78,4$ & 14 & 68,0 & $65,7-70,4$ & 14 \\
\hline Manaus & 90,3 & $86,9-92,9$ & 7 & 82,2 & $78,8-85,1$ & 8 & 74,3 & $71,1-77,2$ & 7 \\
\hline Boa Vista & 87,2 & $84,1-89,7$ & 12 & 79,7 & $77,0-82,2$ & 12 & 73,2 & $70,7-75,6$ & 10 \\
\hline Belém & 94,4 & $92,1-96,0$ & 3 & 89,6 & $87,2-91,6$ & 2 & 84,6 & $82,3-86,7$ & | \\
\hline Macapá & 95,1 & $93,7-96,2$ & । & 90,0 & $88,4-91,5$ & । & 84,5 & $82,8-86,0$ & 2 \\
\hline Palmas & 92,7 & $90,9-94,2$ & 6 & 84,6 & $82,4-86,6$ & 6 & 75,8 & $73,6-77,9$ & 6 \\
\hline Região Nordeste & 85,9 & $85,1-86,7$ & . & 74,8 & $74,0-75,6$ & . & 64,8 & $64,0-65,6$ & . \\
\hline São Luís & 93,5 & $91,4-95,1$ & 4 & 87,1 & $84,8-89,1$ & 3 & 80,4 & $78,1-82,5$ & 3 \\
\hline Teresina & 92,7 & $90,8-94,1$ & 6 & 86,4 & $84,5-88,2$ & 5 & 80,1 & $78,2-81,9$ & 4 \\
\hline Fortaleza & 94,4 & $93,1-95,4$ & 2 & 83,2 & $81,3-85,0$ & 7 & 68,7 & $66,6-70,8$ & 13 \\
\hline Natal & 83,0 & $79,9-85,7$ & 18 & 71,2 & $68,5-73,8$ & 19 & 61,2 & $58,5-63,7$ & 19 \\
\hline João Pessoa & 79,6 & $76,6-82,2$ & 24 & 67,2 & $64,7-69,5$ & 24 & 57,1 & $54,9-59,3$ & 23 \\
\hline Recife & 83,2 & $79,1-86,6$ & 17 & 69,5 & $65,9-72,9$ & 22 & 57,6 & $54,3-60,9$ & 22 \\
\hline Maceió & 78,1 & $75,3-80,7$ & 25 & 64,6 & $62,3-66,9$ & 26 & 53,8 & $51,7-55,9$ & 26 \\
\hline Aracaju & 82,5 & $79,7-85,0$ & 19 & 70,9 & $68,5-73,2$ & 20 & 61,2 & $59,0-63,3$ & 18 \\
\hline Salvador & 84,7 & $81,5-87,4$ & 15 & 74,4 & $71,6-77,0$ & 16 & 65,4 & $62,9-67,9$ & 16 \\
\hline RegiãoSudeste & 83,5 & $81,9-85,0$ & . & 72,3 & $70,9-73,7$ & $\cdots$ & 62,6 & $61,3-63,9$ & . \\
\hline Belo Horizonte & 82,4 & $79,5-84,9$ & 20 & 69,9 & $67,4-72,3$ & 21 & 59,4 & $57,1-61,6$ & 21 \\
\hline Vitória & 87,6 & $85,2-89,6$ & $\|$ & 78,8 & $76,6-80,9$ & 13 & 70,9 & $68,8-72,9$ & 12 \\
\hline São Paulo & 79,7 & $76,2-82,8$ & 23 & 66,2 & $63,3-69,0$ & 25 & 55,2 & $52,7-57,8$ & 25 \\
\hline RegiãoSul & 82,5 & $80,6-84,3$ & $\cdots$ & 70,7 & $69,0-72,4$ & $\cdots$ & 60,8 & $59,2-62,3$ & $\cdots$ \\
\hline Curitiba & 83,5 & $80,7-86,0$ & 16 & 71,4 & $68,8-73,8$ & 18 & 60,9 & $58,6-63,1$ & 20 \\
\hline Florianópolis & 82,2 & $78,9-85,2$ & 21 & 71,6 & $68,7-74,3$ & 17 & 62,6 & $60,1-65,1$ & 17 \\
\hline Porto Alegre & 81,3 & $76,7-85,2$ & 22 & 68,0 & $64,2-71,7$ & 23 & 56,9 & $53,4-60,4$ & 24 \\
\hline Região Centro-Oeste & 90,2 & $89,3-91,0$ & 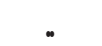 & 81,6 & $80,6-82,5$ & . & 73,1 & $72,2-74,1$ & . \\
\hline Campo Grande & 89,9 & $87,6-91,9$ & 8 & 81,0 & $78,6-83,2$ & $\|$ & 72,3 & $70,0-74,5$ & II \\
\hline Cuiabá & 89,6 & $87,4-91,4$ & 9 & 81,2 & $79,1-83,2$ & 9 & 73,3 & $71,2-75,2$ & 9 \\
\hline Goiânia & 85,9 & $83,5-87,9$ & 13 & 75,3 & $73,1-77,4$ & 15 & 65,8 & $63,7-67,7$ & 15 \\
\hline Distrito Federal & 93,4 & $92,2-94,5$ & 5 & 86,4 & $84,9-87,8$ & 4 & 78,8 & $77,2-80,2$ & 5 \\
\hline Brasil & 87,3 & $86,8-87,7$ & . & 77,5 & $77, \mid-78,0$ & . & 68,6 & $68,2-69,1$ &. \\
\hline
\end{tabular}

*Aordem de apresentaçãa das unidades da Federaçãoe é por critério geográfico, como habitualmente são apresentadas as estimativas de saúde no Brasil.

† Os valores representam os intervalos de confiança (IC) de 95\% (as margens de erro das estimativas).

‡Osvalores representama ordem de classificação da maior prevalência paraa menor.

de alimento inclusive o leite não-humano. No aleitamento materno exclusivo, requer-se o consumo somente de leite materno.

A análise dos dados compreendeu a obtenção das estimativas de prevalência, por ponto e por intervalo (os intervalos de confiança de $95 \%$ ), para as duas modalidades de aleitamento. Os resultados foram primeiro computados para as capitais. Os cálculos para as regiões brasileiras e para o Brasil estão baseados nas informaç̧ões das capitais.

Todas as estimativas foram feitas utilizando-se a análise de regressão, com o uso do programa estatístico SAS'10. O modelo adotado no processo de estimação das prevalências de aleitamento materno e de aleitamento materno exclusivo foi: $\log \left(p_{i} /\left(1-p_{i}\right)=b_{0}\right.$ $+b_{1}$ (ponto médio da faixa etária). No modelo, $p_{\mathrm{i}}$ é a prevalência na faixa etária i e bo e b, são parâmetros que indicam a associação entre $\mathrm{o}$ aleitamento materno e o aleitamento materno exclusivo nas faixas etárias de 30, 120 e 180 dias. A razão $p_{i} /\left(\mid-p_{i}\right)$ é a chance de uma criança na faixa etária i estar em aleitamento materno e aleitamento materno exclusivo ${ }^{11,12}$.

\section{Resultados}

\section{Estimativa das prevalências}

Os achados são primeiro apresentados para o aleitamento materno e, depois, para o aleitamento materno exclusivo. Para cada idade são mostradas três informações: a prevalência (\%), o intervalo de confiança (ou seja, a margem de erro das estimativas de prevalência) e a ordem de classificação da localidade.

\section{Aleitamento materno}

A Tabela I apresenta as estimativas das prevalências do aleita- 
Tabela 2- Prevalência (\%) de aleitamento materno exclusivo, por idade da criança, nas capitais, Distrito Federal, grandes regiões e estimativa para o Brasil, 1999

\begin{tabular}{|c|c|c|c|c|c|c|c|c|c|}
\hline \multirow[t]{3}{*}{ Localidades* } & \multicolumn{9}{|c|}{ Idade da criança em dias } \\
\hline & \multicolumn{3}{|c|}{30} & \multicolumn{3}{|c|}{120} & \multicolumn{3}{|c|}{180} \\
\hline & $\%$ & IC 95\% ${ }^{\dagger}$ & ordem $\neq$ & $\%$ & $1 \mathrm{C} 95 \%^{\dagger}$ & ordem $\ddagger$ & $\%$ & $1 \mathrm{C} 95 \%{ }^{\dagger}$ & ordem ${ }^{\ddagger}$ \\
\hline Região Norte & 47,0 & $44,7-49,3$ &. & 16,8 & $15,6-18,0$ & . & 7,0 & $6,1-8,1$ &. \\
\hline Porto Velho & 35,1 & $29,7-41,0$ & 19 & 11,7 & $9,3-14,5$ & 21 & 4,9 & $3,2-7,5$ & 21 \\
\hline Rio Branco & 35,8 & $30,0-42,0$ & 18 & 9,7 & $7,5-12,5$ & 25 & 3,5 & $2,1-5,6$ & 25 \\
\hline Manaus & 31,4 & $24,0-39,9$ & 24 & 12,2 & $9,0-16,3$ & 20 & 5,9 & $3,3-10,3$ & 17 \\
\hline Boa Vista & 42,9 & $36,6-49,4$ & 15 & 14,0 & $11,2-17,5$ & 15 & 5,6 & $3,6-8,6$ & 18 \\
\hline Belém & 63,1 & $55,7-69,9$ & 4 & 32,2 & $28,1-36,6$ & । & 16,9 & $12,4-22,6$ & । \\
\hline Macapá & 61,1 & $56,5-65,5$ & 5 & 21,8 & $18,9-24,9$ & 7 & 8,1 & $6,0-10,8$ & 11 \\
\hline Palmas & 48,0 & $42,7-53,4$ & 12 & 15,6 & $13,0-18,7$ & 14 & 6,0 & $4,1-8,5$ & 15 \\
\hline Região Nordeste & 49,9 & $48,|-5|, 7$ &. & 19,3 & $18,3-20,3$ & . & 8,4 & $7,6-9,4$ &. \\
\hline São Luís & 55,7 & $49,3-61,9$ & 6 & 28,5 & $24,8-32,4$ & 4 & 15,6 & $11,6-20,8$ & 2 \\
\hline Teresina & 55,0 & $49,8-60,1$ & 7 & 22,0 & $|9|-25,, \mid$ & 6 & 9,6 & $7,2-12,7$ & 7 \\
\hline Fortaleza & 73,4 & $69,6-76,9$ & | & 29,0 & $26,1-32,0$ & 3 & 10,2 & $8,0-13,0$ & 6 \\
\hline Natal & 51,0 & $45,8-56,2$ & 10 & 19,8 & $16,7-23,4$ & 9 & 8,7 & $6,2-12,1$ & 8 \\
\hline João Pessoa & 44,0 & $38,8-49,4$ & 14 & 12,9 & $10,7-15,5$ & 17 & 4,6 & $3,2-6,6$ & 22 \\
\hline Recife & 34,1 & $27,|-4|, 8$ & 20 & 17,3 & $|4,0-2|, 4$ & 13 & 10,3 & $6,7-15,5$ & 5 \\
\hline Maceió & 31,9 & $27,6-36,4$ & 22 & 13,0 & $10,9-15,4$ & 16 & 6,5 & $4,7-9,0$ & 13 \\
\hline Aracaju & 47,3 & $42,3-52,4$ & 13 & 17,6 & $15,0-20,4$ & | & 7,5 & $5,5-10,2$ & 12 \\
\hline Salvador & 37,8 & $31,9-44,2$ & 17 & 12,5 & $9,9-15,6$ & 18 & 5,1 & $3,3-7,9$ & 19 \\
\hline RegiãoSudeste & 38,2 & $35,2-41,3$ & . & 14,5 & $|3,0-16|$, & 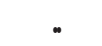 & 6,7 & $5,4-8,1$ & . \\
\hline BeloHorizonte & 30,6 & $26,0-35,6$ & 25 & 10,4 & $8,4-12,9$ & 24 & 4,6 & $3,0-6,8$ & 23 \\
\hline Vitória & 50,1 & $44,9-55,3$ & 11 & 19,6 & $17,0-22,5$ & 10 & 8,6 & $6,5-11,5$ & 9 \\
\hline São Paulo & 32,9 & $27,5-38,7$ & 24 & 12,4 & $10,0-15,4$ & 19 & 5,9 & $3,9-8,8$ & 16 \\
\hline RegiãoSul & 58,5 & $54,9-62,0$ & . & 23,8 & $21,7-26,0$ & $\cdots$ & 10,2 & $8,5-12,4$ & . \\
\hline Curitiba & 55,0 & $49,4-60,5$ & 8 & 20,6 & $17,7-23,8$ & 8 & 8,4 & $6,2-11,4$ & 10 \\
\hline Florianópolis & 66,7 & $60,8-72,1$ & 2 & 31,7 & $27,9-35,7$ & 2 & 14,9 & $11,2-19,5$ & 3 \\
\hline Porto Alegre & 52,7 & $44,9-60,4$ & 9 & 17,4 & $|3,6-22|$, & 12 & 6,5 & $3,9-10,6$ & 14 \\
\hline Região Centro-Oeste & 44,4 & $42, \mid-46,8$ & . & 15,2 & $|4|-,\mid 6,5$ & . & 6,2 & $5,3-7,3$ &. \\
\hline Campo Grande & 40,1 & $34,6-45,9$ & 16 & 10,8 & $8,6-13,6$ & 23 & 3,8 & $2,4-5,9$ & 24 \\
\hline Cuiabá & 25,2 & $20,8-30,2$ & 26 & 7,2 & $5,5-9,4$ & 26 & 2,8 & $1,7-4,6$ & 26 \\
\hline Goiânia & 31,5 & $27,1-36,3$ & 23 & 11,0 & $9,1-13,3$ & 22 & 4,9 & $3,4-7,0$ & 20 \\
\hline Distrito Federal & 63,7 & $60,1-67,2$ & 3 & 25,5 & $23,1-28,0$ & 5 & 10,3 & $8,3-12,7$ & 4 \\
\hline Brasil & 47,5 & $46,4-48,5$ &. & 17,7 & $17,2-18,3$ & & 7,7 & $7,2-8,2$ &. \\
\hline
\end{tabular}

* A ordem de apresentaçãa das unidades da Federação é por critério geográfico, como habitualmente são apresentadas as estimativas de saúde no Brasil.

${ }^{\dagger}$ Os valores representam os intervalos de confiança(IC) de 95\% (as margens de erro das estimativas).

‡Osvalores representam a ordem de classificação da maior prevalência paraa menor.

mento materno. Os dados para o Brasil mostram que a maioria das crianças (87,3\%) é amamentada no primeiro mês de vida. Essa proporção decresce para $77,5 \%$ aos 120 dias, e para $68,6 \%$ aos 180 dias. Os maiores percentuais de prevalência são encontrados nas regiões Norte e Centro-Oeste nas diferentes idades.

\section{Aleitamento materno exclusivo}

A Tabela 2 mostra as estimativas das prevalências do aleitamento materno exclusivo. Para o País, o percentual de crianças alimentadas exclusivamente com leite materno é baixo já no primeiro mês de vida (47,5\%). Na idade de 120 dias, a proporção estimada foi $17,7 \%$ e, aos 180 dias, 7,7\%. A região Sul destaca-se com as maiores prevalências para todas as idades.

Em termos relativos, comparando-se as taxas aos 30 dias e aos 180 dias de vida, houve redução de $21 \%$ na prevalência de aleitamento materno e de $84 \%$ na de aleitamento materno exclusivo.

\section{Distribuição das prevalências}

Os diagramas de caixa mostram as características da distribuiçãa das freqüências dos aleitamentos materno e exclusivo nas idades analisadas (Figura I). Para o aleitamento materno, as taxas são elevadas e a dispersão entre as idades pouco difere. Referentemente ao aleitamento materno exclusivo, evidencia-se o nítido decréscimo das prevalências no primeiro semestre de vida. Observa-se também maior heterogeneidade das taxas aos 30 dias e tendência para a uniformidade com valores bastantes baixos aos 180 dias.

\section{Discussão}

O presente estudo informa a distribuição etária e geográfica da 


\section{Figura I - Distribuição das prevalências do aleitamento materno e do aleitamento exclusivo nas capitais brasileiras, conforme idade da criança, 1999 ( $n=26$ unidades da Federação)}

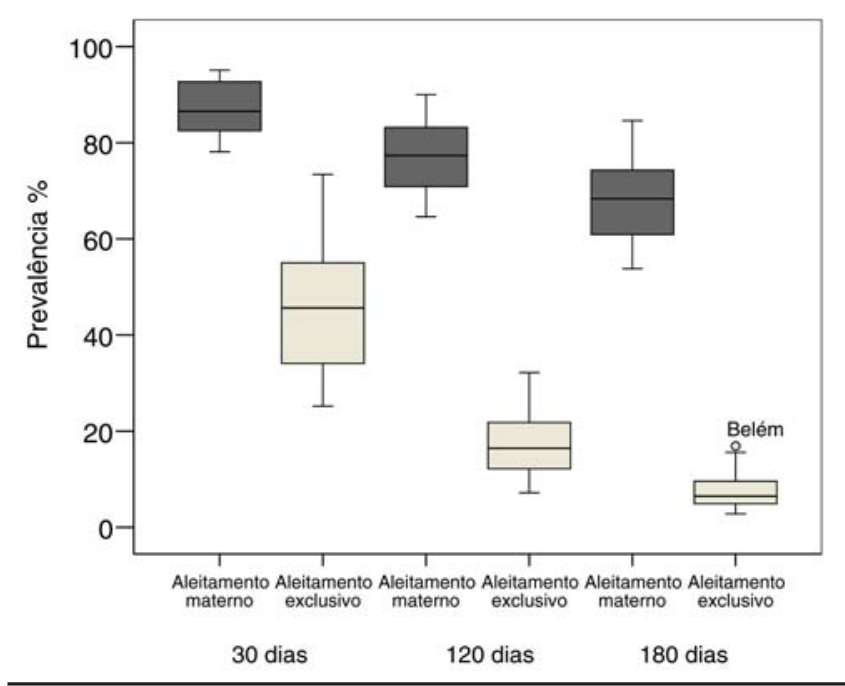

freqüência de amamentação de crianças com até seis meses de idade na área urbana das capitais brasileiras e as estimativas para as regiões e para o País. Em relação à idade, houve redução gradual da prevalência do aleitamento materno (19\%) e queda acentuada da prevalência do aleitamento materno exclusivo (39,8\%). Também foram observadas diferenças expressivas na freqüência do aleitamento materno exclusivo entre as capitais pesquisadas.

○ padrão observado, de declínio da amamentação nos primeiros seis meses de vida, é semelhante ao verificado em dois estudos nacionais prévios de grande porte, a PNSN 5 e a PNDS 6 , realizadas em 1989 e 1996, respectivamente. Ressalte-se o aumento das prevalências no período.

Algumas diferenças e semelhanças na freqüência do aleitamento materno exclusivo entre as capitais pesquisadas merecem ser realçadas (Tabela 2). Em Fortaleza, por exemplo, os percentuais de crianças com 30 e 120 dias de vida alimentadas somente com o leite materno correspondem a 73,4\% e 29\%, respectivamente. Em Recife, as taxas de amamentação, nas mesmas idades, situam-se em 34, 1\% e 17,3\%. No entanto, aos 180 dias, ambas as localidades apresentam prevalências semelhantes dessa modalidade de aleitamento: Fortaleza com 10,2\% e Recife com 10,3\%. Os resultados sugerem que as capitais em melhor situação de aleitamento materno exclusivo nos primeiros meses de vida encontram dificuldades em manter essa condição e chegam no fim do primeiro semestre de vida em patamares semelhantes aos verificados nos municípios que não alcançaram desempenho tão favorável no início do período.

Comportamento semelhante foi observado ao se comparar a ordem de classificação das capitais nos dois tipos de aleitamento. Melhor desempenho em relação ao aleitamento materno não implica necessariamente a mesma posição em relação à amamentação exclusiva, ou vice-versa, como observado em Macapá e Florianópolis. As diferentes condutas dos grupos populacionais em relação à amamentação são moduladas, em sua maioria, por preferências pessoais, culturais, circunstâncias sociais e econômicas, características demográficas ${ }^{13-17}$ e aplicação de programas e ações voltados para evitar o desmame precoce ${ }^{18-20} .0$ Brasil, por ser muito extenso territorialmente, é diversificado em relação aos aspectos mencionados, por isso, as variações de aleitamento natural observadas. A heterogeneidade de prevalências evidenciada neste inquérito indica que a influência desses fatores no desmame varia com a idade e tem peso distinto em cada contexto.

Embora haja evidências da melhora da situação do aleitamento materno entre as crianças brasileiras, ${ }^{21,22}$ a situação no País em relação à amamentação exclusiva é preocupante. $O$ percentual de crianças no primeiro semestre de vida, alimentadas somente com - leite materno, permanece muito aquém da recomendação do Unicef, da OMS e do Ministério da Saúde ${ }^{1,2}$. O cenário mundial em relação a essa modalidade de amamentação também se mostra desfavorável. Nos anos 90, o aumento na freqüência da amamentação exclusiva em menores de quatro meses, de $48 \%$ para 52\%, foi considerado modesto ${ }^{23}$. A análise envolveu 37 países em desenvolvimento. Na América Latina e no Caribe, os ganhos foram de maior destaque. Contudo, a região permanece com os menores valores em relação a todos os indicadores de amamentação.

\section{ConClusão}

O perfil de amamentação na área urbana do País pode ser considerado satisfatório para o aleitamento materno e preocupante para o aleitamento materno exclusivo. Algumas faixas etárias e capitais apresentam prevalências muito baixas. É necessário concentrar esforços na aplicação de medidas de promoção e de proteção ao aleitamento natural. Ressalte-se ainda que a padronização metodológica, adotada neste inquérito sobre amamentação no Brasil, permite direta comparação geográfica e cronológica.

\section{Conflito de interesse: não há.}

\section{SUMMARY}

\section{Prevalence of breastfeeding in Brazilian capital cities}

OBJECTIVE. To estimate the prevalence of breastfeeding and of exclusive breastfeeding in Brazilian capital cities, in the 5 major geographical areas of Brazil and in the whole country, at the ages of 30, 120 and 180 days, as agreed among specialists.

METHODS. Restudy of data from the population inquiry about breastfeeding in 25 capital cities and in the Federal District during a mass immunization campaign, on October 16 th , 1999, National Day of Vaccination, supervised by one of the authors. The random sample of this study refers to 10,778 children, according to the ages mentioned above. The point and interval estimates (95\% Cl) were given for the capital cities and then extrapolated to the major geographical areas and to Brazil. The regression analysis was used on the SAS statistical program. 
RESULTS. The estimated prevalence of breastfeeding in Brazil was 87.3\% (Cl 95\%: 86.8 - 87.7) at the age of 30 days, $77.5 \%$ (77.1 - 78.0) at the age of 120 days and $68.6 \%(68.2-69.1)$ at the age of 180 days. The exclusive breastfeeding prevalence was $47.5 \%$ (46.4 - 48.5), $17.7 \%(17.2-18.3)$ and $7.7 \%(7.2-8.2)$ at the ages mentioned. At the age of 30 days, variation of the frequency of exclusive breastfeeding was wide, from $73.4 \%$ (Fortaleza) to $25.2 \%$ (Cuiaba). At the age of 180 days, the prevalence ranged from 16.9\% in Belem to $2.8 \%$ in Cuiaba.

CONCLUSION. There was a moderate reduction of the prevalence of breastfeeding and a steep decline of the prevalence of exclusive breastfeeding from birth to the age of 180 days. Important differences were noted in the frequency of exclusive breastfeeding among the capital cities surveyed. [Rev Assoc Med Bras 2007; 53(6): $520-4]$

KEY WORDS: Breastfeeding. Exclusive breastfeeding. Cross-sectional studies. Epidemiology. Brazil

\section{REFERÊNCIAS}

I. Kramer MS, Kakuma R. The optimal duration of exclusive breastfeeding: a systematic review. Switzerland: World Health Organization; 2002.

2. Monte CMG, Giugliani ERJ, Carvalho MFCC, Philippi ST, Albuquerque ZP. Guia alimentar para crianças menores de 2 anos. Brasília: Ministério da Saúde; 2002. (Série A. Normas e Manuais Técnicos, n. 107).

3. Pereira MG. Epidemiologia: teoria e prática. Rio de Janeiro: Guanabara Koogan; 2000. p.270-417.

4. Notzon F. Trends in infant feeding in developing countries. Report of the task force on the scientific evidence relating to infant-feeding practices and infant health. Pediatrics. 1984;74(4 Suppl): S648-66.

5. Leão MM, Coitinho DC, Recine E, Costa LAL, Lacerda AJ. O Perfil do aleitamento materno no Brasil. In: Monteiro MFG, Cervini R, organizadores. Perfil estatístico de crianças e mães no Brasil: aspectos de saúde e nutrição de crianças no Brasil 1989. Rio de Janeiro: IBGE/ Departamento de Estatísticas e Indicadores Sociais; 1992. p.97-109

6. Amamentação e situação nutricional de mães e crianças. In: Brasil. Ministério da Saúde. Pesquisa nacional sobre demografia e saúde 1996. $2^{\mathrm{a}}$ ed. Rio de Janeiro: BEMFAM / Programa de Pesquisas de Demografia e Saúde DHS; 1999. p.125-38.

7. Ministério da Saúde. Secretaria de Políticas de Saúde. Prevalência do aleitamento materno nas capitais brasileiras e no Distrito Federal. Brasília (DF); $200 \mathrm{I}$.

8. Rede Interagencial de Informações para a Saúde- RIPSA. Indicadores básicos para a saúde no Brasil: conceitos e aplicações. Brasília (DF): Organização Pan-Americana da Saúde; 2002.
9. Indicators for assessing breastfeeding practices. Geneva: World Health Organization; 1991.

10. SAS Institute Inc., SAS/STAT ${ }^{\circledR}$ Software: Changes and Enhancements, release 8.2. Cary: SAS Institute Inc.; 200I.

I I. Sena MCF. Prevalência do aleitamento materno exclusivo no Distrito Federal e sua associação com o trabalho materno fora do lar [dissertação]. Brasília (DF): Faculdade de Ciências da Saúde, Universidade de Brasília; 1997.

12. Sena MCF. O aleitamento materno no Distrito Federal nos anos 90. Brasília (DF): Fundação de Ensino e Pesquisa em Ciências da Saúde; 2002.

13. Grummer-Strawn LM. The effect of changes in population characteristics on breastfeeding trends in fifteen developing countries. Int J Epidemiol. | 996;25:94-101.

14. Pérez-Escamilla R, Lutter C, Segall AM, Rivera A, Trevino-Siller S, Sanghvi T. Exclusive breast-feeding duration is associated with attitudinal, socioeconomic and biocultural determinants in three Latin American Countries. J Nutr. 1995; 125:2972-84.

15. Huffman SL. Determinants of breastfeeding in developing countries: overview and policy implications. Stud Fam Plann. 1984; I 5: 170-83.

16. Giugliani ERJ, Issler RMS, Justo EB, Seffrin CF, Hartmann RM, Carvalho NM. Risk factors for early termination of breast feeding in Brazil. Acta Paediatr. 1992:81:484-7.

17. Bueno MB, Souza JMP, Souza SB, Paz SMRS, Gimeno SGA, Siqueira AAF. Riscos associados ao processo de desmame entre crianças nascidas em hospital universitário de São Paulo, entre 1998 e 1999: estudo de coorte prospectivo do primeiro ano de vida. Cad Saúde Pública. 2003; 19:1453-9.

18. Venâncio SI, Monteiro CA. Individual and contextual determinants of exclusive breast-feeding in São Paulo, Brazil: a multilevel analysis. Public Health Nutr. 2005;9:40-6.

19. Coutinho SB, Lira PIC, Lima MC, Ashworth A. Comparison of the effect of two systems for the promotion of exclusive breastfeeding. Lancet. 2005;366: 1094-100.

20. Marques NM, Lira PIC, Lima MC, Silva NL, Batista Filho M, Sharon RA, et al. Breastfeeding and early weaning practices in northeast Brazil: a longitudinal study. [cited 2007 may]. Pediatrics. 200I; 108(4). Avaliable from: http://www.pediatrics.org/cgi/content/full/I08/4/e66.

21 . Venâncio SI, Monteiro CA. A tendência da prática da amamentação no Brasil nas décadas de 70 e 80. Rev Bras Epidemiol. 1998; 1:40-9.

22. Pérez-Escamilla R. Breastfeeding and the nutritional transition in the Latin American and Caribbean Region: a success story? Cad Saúde Pública. 2003;19(Suppl I):S119-27.

23. Breastfeeding and complementary feeding. Unicef Statistics; ( 6 ). [cited 2006 jul 3]. Avaliable from: http://childinfo.org/eddb/brfeed/index.htm.

Artigo recebido: I //05/07

Aceito para publicação: 08/08/07 